\title{
Heinz Bodies Induce Clustering of Band 3, Glycophorin, and Ankyrin in Sickle Cell Erythrocytes
}

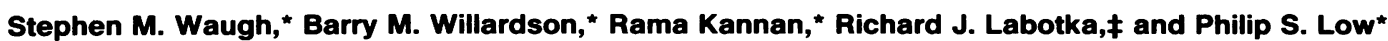

*Department of Chemistry, Purdue University, West Lafayette, Indiana 47907; and $\ddagger$ Department of Pediatrics, College of Medicine, University of Illinois at Chicago, Chicago, Illinois 60612

\begin{abstract}
In earlier model studies we demonstrated that artificially denatured hemoglobin binds to and clusters the protein, band 3, in the plane of the erythrocyte membrane. To determine whether denatured hemoglobin also clusters band 3 in vivo, we have compared the locations of denatured hemoglobin aggregates (Heinz bodies) with band 3 in sickle cells using phase contrast and immunofluorescence microscopy. We report that where Heinz bodies are found associated with the cytoplasmic surface of the membrane, clusters of band 3 are usually colocalized within the membrane. In contrast, normal erythrocyte membranes and regions of sickle cell membranes devoid of Heinz bodies display an uninterrupted staining of band 3. Similarly, ankyrin and glycophorin are periodically seen to aggregate at Heinz body sites, but the degree of colocalization is lower than for band 3 . These data demonstrate that the binding of denatured hemoglobin to the membrane forces a redistribution of several major membrane components.
\end{abstract}

\section{Introduction}

Erythrocytes containing unstable or mutant hemoglobins commonly exhibit an enhanced tendency to release membrane vesicles and/or to hemolyze $(1,2)$. Because the amino acid change in these hemoglobins is believed to be the sole primary defect in the cells, the associated membrane abnormalities must derive either directly or indirectly from the altered properties of the mutant hemoglobins. In the case of sickle cells, hypotheses relating the molecular defect in the hemoglobin to the altered properties of the membrane e.g., flexibility, $\mathrm{Ca}^{2+}$ permeability, lipid asymmetry, cytoskeletal structure, surface charge distribution, autologous antibody affinity, etc. (3-14), have generally attributed the membrane lesions either to the tendency of sickle hemoglobin $(\mathrm{HbS})^{1}$ to polymerize and distort the cell, or to its predisposition to denature and catalyze the production of oxidizing agents. In the latter hypothesis, the oxidizing agents are believed to react with membrane proteins and/or lipids in causing the defective membrane behavior (15).

In recent model studies, we have presented evidence that slightly denatured hemoglobins, termed hemichromes, associate tightly with the cytoplasmic domain of the predominant mem-

Address correspondence to Dr. Philip S. Low.

Received for publication 19 December 1985 and in revised form 14 July 1986

1. Abbreviations used in this paper: $\mathrm{HbS}$, sickle hemoglobin.

J. Clin. Invest.

(c) The American Society for Clinical Investigation, Inc. 0021-9738/86/11/1155/06 \$1.00

Volume 78, November 1986, 1155-1160 brane-spanning protein, band 3, both in situ and in isolated form (16). Importantly, this interaction was shown to be of much higher affinity than the association of native hemoglobin with band 3 (16). Furthermore, the tight association between hemichromes and band 3 was found not to terminate in a simple unit complex, but instead to propagate into a stoichiometric copolymer of macromolecular dimensions (16). When the model study was extended to include intact cells, it was found that phenylhydrazine-generated hemichromes bound tightly to the cytoplasmic surface of the membrane, promoting a redistribution of band 3 in situ (17). Although no investigation of hemichromemembrane interactions in unmodified naturally occurring cells was ever attempted, it was hypothesized that a similar hemichrome-induced clustering of band 3 in the membrane might be observed wherever hemichromes aggregate on the membrane surface.

To evaluate the above hypothesis, we have examined the lateral distribution of band 3, ankyrin, and glycophorin in sickle cells by immunofluorescence microscopy. Our results demonstrate that at most sites where hemichrome aggregates (Heinz bodies) are firmly attached to the membrane, band 3 is also clustered within the membrane. Ankyrin and glycophorin are also frequently observed to colocalize with Heinz body sites on the membrane, however the correlation in these cases is not as great as is observed for band 3 .

\section{Methods}

Antibodies. Human erythrocyte ankyrin was prepared according to Bennett and Stenbuck (18). This preparation was applied to a preparative sodium dodecyl sulfate polyacrylamide gel and the major band at 210,000 -mol wt was cut from the gel, emulsified, and used to raise antiankyrin antibodies in rabbits. The antiserum from these rabbits was found by immunoblotting to stain exclusively ankyrin and its degradation products, band 2.1-2.6 (Fig. 1, lane $A$ ). No staining of isolated spectrin was observed with this preparation. Antibodies were also made against the cytoplasmic domain of band 3 by injecting rabbits with the native protein prepared according to reference 19 as modified in reference 20 . The anti-band 3 antiserum was affinity-purified on peripheral proteindepleted inside-out vesicles that were prepared by low ionic strength extraction of red cell membranes (21) followed by stripping with $1 \mathrm{M}$ $\mathrm{KCl}(22)$. The antibodies were eluted from the vesicles with $0.1 \mathrm{M}$ glycine buffer, $\mathrm{pH} 2.7$, and were found on western blots of red cell ghosts to stain exclusively band 3 (Fig. 1, lane $B$ ).

The IgG fraction of the antiserum raised in goat against rabbit IgG and labeled with rhodamine isothiocyanate was obtained from Sigma Chemical Co., St. Louis, MO, as a $17.1-\mathrm{mg} / \mathrm{ml}$ stock solution with a molar ratio of rhodamine to protein of 3.0. The biotin-conjugated goat anti-rabbit and goat anti-human IgG and the rhodamine-conjugated avidin were also from Sigma Chemical Co.

Sickle cells. Red blood cells were obtained from 19 pediatric and adult patients (age range 3-53 yr) with major sickle hemoglobinopathies (15 Hb SS, $1 \mathrm{Hb} \mathrm{S} \beta^{\circ}$-thalassemia, 3HbSC) being followed at the University of Illinois Comprehensive Sickle Cell Center. Diagnoses were 


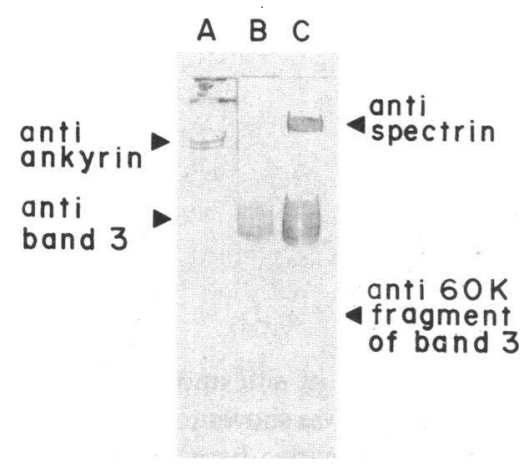

Figure 1. Specificities of antibody preparations to ankyrin and the cytoplasmic domain of band 3. Erythrocyte ghosts in sodium dodecyl sulfate were separated electrophoretically on $5.5 \%$ polyacrylamide gels according to the procedure of Fairbanks et al. (40), blotted onto nitrocellulose paper, and labeled with antiserum to ankyrin (lane $A$ ), affinity-purified antibody to the cytoplasmic domain of band 3 (lane $B$ ), and a mixture of antibodies to spectrin and band 3 (lane $C$ ). After treatment with the above primary antibodies, the blots were washed and the labeled polypeptides were visualized by staining with perioxidase-conjugated goat anti-rabbit IgG and 4-chloronaphthol.

based upon hemogram, Wright-stained peripheral blood smear, positive sickle solubility test, and cellulose acetate hemoglobin electrophoresis at pH 8.6. After consent, 3-5 ml of venous blood were collected into EDTA, when cells were to be used immediately, or acid citrate dextrose-containing vacutainer tubes. Specimens were transported on ice and refrigerated until use. Packed red cells were prepared by centrifugation and removal of supernatant plasma and buffy coat.

Immunostaining of red cells. Red cells were washed several times in phosphate-buffered saline (PBS) (20 mM sodium phosphate, $125 \mathrm{mM}$ $\mathrm{NaCl}, \mathrm{pH} 7.4$ ) and diluted to $50 \%$ hematocrit with PBS containing $4 \%$ bovine serum albumin (BSA). Blood smears were then prepared and allowed to dry at room temperature for at least $1 \mathrm{~h}$. Typically, air-dried smears were made within 2-3 d of blood collection and used immediately. However, in three cases the smears were examined immediately after blood letting. There were no major differences in the results from the fresh and briefly stored blood samples.

Before being immunostained, the air-dried smears were permeabilized and fixed with acetone for $2 \mathrm{~min}$ at room temperature and then washed three times with PBS, pH 7.4. For ankyrin and band 3 staining, small areas of the smears were overlaid with a 1:30 dilution of ankyrin immune serum or affinity-purified antibodies against the cytoplasmic domain of band 3 ( $33 \mu \mathrm{g} \mathrm{IgG/ml,} \mathrm{final)} \mathrm{diluted} \mathrm{into} \mathrm{PBS,} \mathrm{pH} \mathrm{7.4,} \mathrm{containing} 0.5 \%$ BSA. After a 30-min incubation, the smears were washed 3 times with PBS and then incubated with a 1:30 dilution of rhodamine-conjugated anti-rabbit IgG. After repeated washing, the stained cells were overlaid with either buffer or $35 \%$ glycerol, sealed under a coverslip, and viewed with a BHT fluorescence microscope (Olympus Corp. of America, New Hyde Park, NY). In experiments designed to localize autologous antibodybinding sites on the erythrocyte cell surface, the smears were either directly stained with rhodamine-conjugated antihuman IgG, or incubated first with biotin-conjugated antibodies $(0.5 \mu \mathrm{g} / \mathrm{ml})$, then with rhodamineconjugated avidin $(5.0 \mu \mathrm{g} / \mathrm{ml})$ after being washed. In these cases, the dilutions were made in PBS containing 4\% BSA.

Direct staining of glycophorin in situ. Glycophorin was specifically labeled with fluorescein thiosemicarbazide according to previously published procedures (23-25). Basically, cell surface sialic acid residues were mildly oxidized in PBS containing $2 \mathrm{mM}$ sodium metaperiodate for $\mathbf{3 0}$ min on ice. The cells were then washed 3 times in PBS and labeled in the same buffer containing $0.2 \mathrm{mg} / \mathrm{ml}$ fluorescein thiosemicarbazide. Although some glycolipid labeling is observed, the vast majority of the fluorescence is found associated with glycophorin, and no fluorescence is detected on band $3(23,25)$. The glycophorin-labeled cells were viewed either in suspension or after smearing and drying as described above.

\section{Results}

Intracellular precipitates of denatured hemoglobin (Heinz bodies) were found in abundance in the red cells of most sickle hemoglobinopathy patients. The two examples shown in Fig. 2 are representative of 17 of the 19 different samples of sickle cells we have examined to date. When viewed by phase-contrast microscopy, the Heinz bodies were seen as dark inclusion bodies within the erythrocyte and often were seen in juxtaposition to the plasma membrane (Fig. 2, $a$ and c). The Heinz bodies could also be readily visualized in sickle cells in suspension upon treatment of the cells with the stain methyl violet (not shown). Most sickle cells were found to contain one or more of these Heinz bodies while normal cells were largely free of any inclusion bodies (Fig. $3 a$ ). Two of the sickle cell samples, one HbSS and the other $\mathrm{HbS} \beta_{\text {thal }}$, contained significantly fewer Heinz bodies: less than one per cell on the average.

In a previous paper we demonstrated that hemichromes, precursors of Heinz bodies, bound with high affinity to the cytoplasmic domain of the integral membrane protein, band 3 (16). Since the association was found to propagate rapidly into a macromolecular copolymer, we proposed that the band 3hemichrome interaction would lead to a lateral clustering of band 3 within the plane of the membrane (17). Sickle cells provided us with an excellent system on which to test our hypothesis, since Heinz bodies are a normal constituent of these cells, which eliminates concerns about artifacts arising from artificially induced Heinz body formation. Affinity-purified antibodies to the cytoplasmic domain of band 3 were used to immunostain erythrocytes that had been permeabilized and fixed with acetone. Normal erythrocytes show a diffuse staining over the entire cytoplasmic surface of the cell, indicating the even distribution of band 3 within the membrane (Fig. $3 \mathrm{~b}$ ). Sickle cells also show staining of the entire surface of the cell, and in addition they display distinct patches of very intense fluorescence staining (Fig. $2, b$ and $d)$. These patches representing dense clusters of band 3 are invariably superimposed on Heinz bodies (compare $a$ with $b$ or $c$ with $d$ in Fig. 2). Usually there is a good correlation between the size and shape of the band 3 cluster and that of the Heinz body. Most Heinz bodies are associated with a band 3 cluster. A Heinz body that does not correlate with a cluster of band 3 molecules can be seen in Fig. $2 a$. A survey of cells from five different donors revealed that on the average $\approx 64 \%$ of the Heinz bodies correlated with a band 3 aggregate (Table I). However, the range of values in this correlation was from 52 to $84 \%$, indicating significant variability among donors. In this respect, we have noticed that the Heinz bodies in some cells are free within the cytoplasm while in other cells the inclusion bodies appear immobilized on the membrane. Obviously, only the latter would be expected to be capable of clustering band 3. Photographs of sickle cells treated with rhodamine-labeled goat anti-rabbit IgG in the absence of the rabbit antibody to band 3 showed no fluorescence when exposed for the same period as the cells stained with anti-band 3.

Immunostaining of normal and sickle cells was also performed using an antibody to human erythrocyte ankyrin. The 

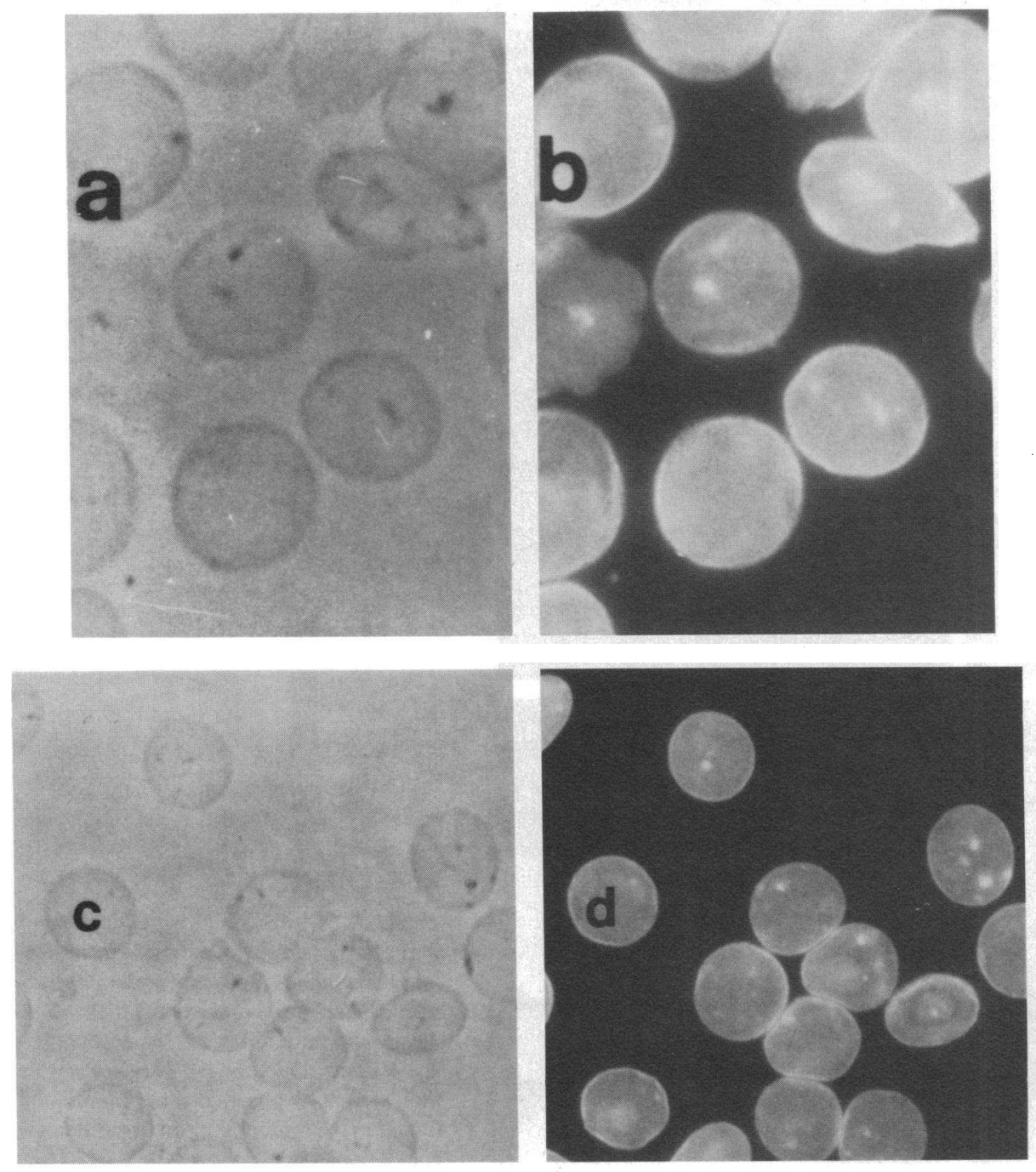

Figure 2. Colocalization of Heinz bodies with clusters of band 3 in erythrocytes from sickle hemoglobinopathy patients. Cells from two individuals were immunostained with affinity-purified antibodies against the cytoplasmic domain of band 3 and viewed by phase contrast microscopy ( $a$ and $c$ ) or immunofluorescence microscopy $(b$ and $d)$. immunostaining of normal red cells was similar to that seen with anti-band 3 , except the fluorescence staining was generally less intense (Fig. 3, $c$ and $d$ ). Sickle cells again showed clusters of ankyrin colocalized with some Heinz bodies (Fig. 4, $a$ and $b$ ). This result is not surprising since ankyrin is tightly associated with band 3 and should co-cluster with the protein $(18,26)$. However, as seen in the figure, the fraction of Heinz bodies coincident with a cluster of ankyrin molecules was less than that observed for band 3; on the average only $37 \%$ showed colocalization (Table I). This observation may be explained if the denatured hemoglobin preferentially coaggregates with the population of band 3, which is not linked to ankyrin (26) and is therefore more free to diffuse. However, other explanations are also possible.

When glycophorin was visualized on sickle cell surfaces by a direct staining procedure (see Methods), a result similar to the behavior of ankyrin was seen (Fig. 5, $a$ and $b$ ). Most, but not all, of the Heinz bodies showed no corresponding fluorescence intensity, suggesting less frequent clustering of glycophorin at Heinz body sites than was observed for band 3 . Of the three sickle cell samples examined by this procedure, 9,25 , and $33 \%$ colocalization with Heinz bodies was observed.

\section{Discussion}

The data presented above provide further support for our hypothesis that Heinz body binding to the membrane promotes the lateral aggregation of band 3 (17). Previous evidence for the Heinz body-induced clustering of band 3 came from two in vitro studies, one where band 3 was shown by biochemical techniques to constitute the high affinity binding site for hemichromes in leaky ghosts, and the other where the isolated, watersoluble cytoplasmic domain of band 3 was shown to copolymerize with hemichromes into a water-insoluble macromolecular complex (16). The colocalization of band 3 clusters with Heinz bodies seen in this study provides the first direct evidence that the macroscopic coaggregation of band 3 and hemichromes observed in vitro also occurs in vivo.

Although the majority of Heinz body sites were found to correlate with band 3 clusters, some Heinz bodies were observed that caused no apparent redistribution of membrane proteins. This variability in the effect of Heinz bodies on membrane organization could arise from three sources. First, some hemichromes undoubtedly aggregate in the cytoplasm and remain there or diffuse as intact Heinz bodies to the membrane. These 

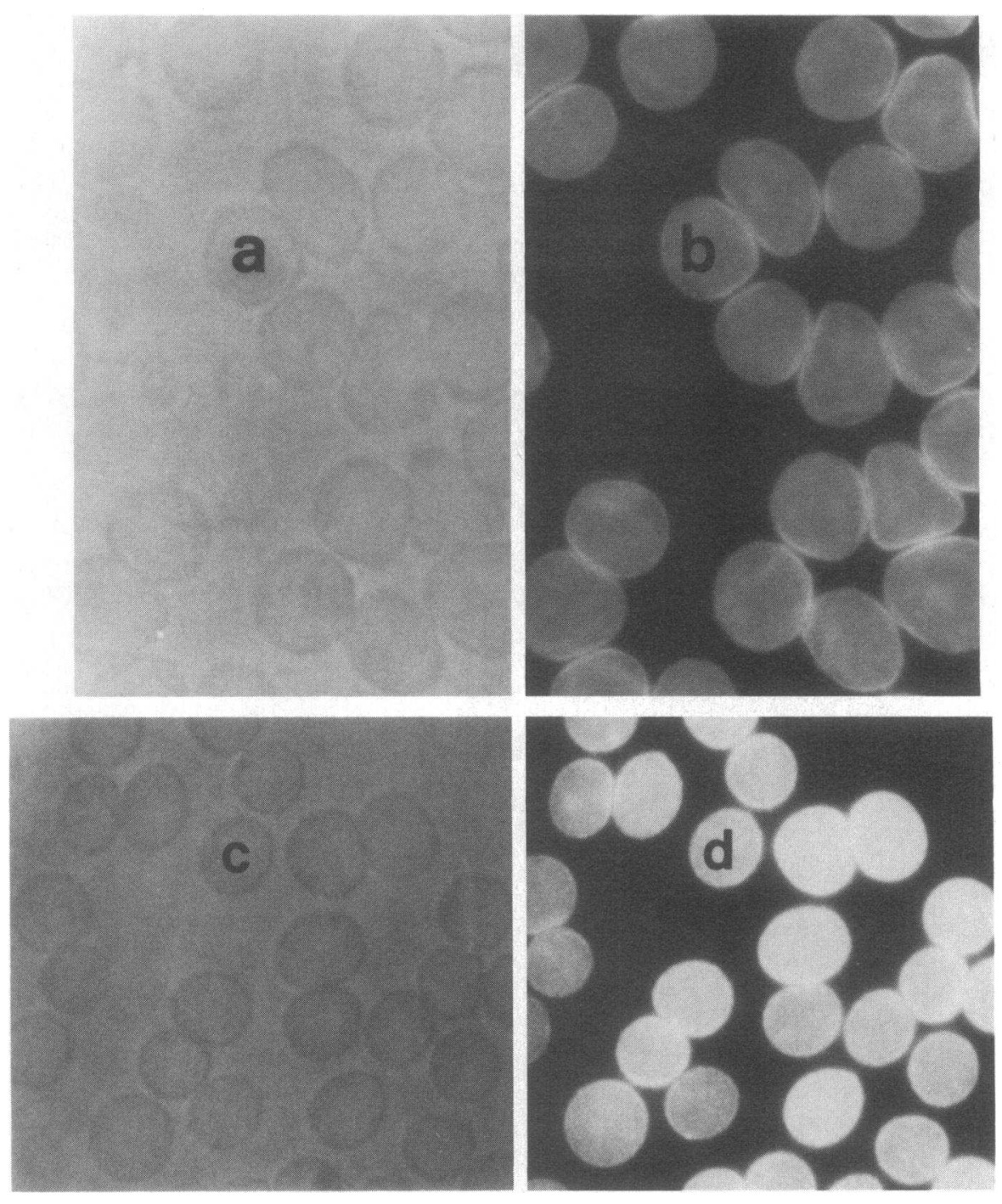

Figure 3. Distribution of band 3 and ankyrin in normal red cells free of intracellular Heinz bodies. Red cells were immunostained with antibodies against the cytoplasmic domain of band $3(a$ and $b$ ) or against ankyrin ( $c$ and $d$ ). These cells were then viewed by phase contrast microscopy ( $a$ and $c$ ) or immunofluorescence microscopy $(b$ and $d)$.
Table I. Percent Colocalization of Anti-band 3 and Anti-ankyrin Immunofluorescence Staining with Sites of Heinz Body Binding to the Membranes of Sickle Cells

\begin{tabular}{lll}
\hline $\begin{array}{l}\text { Sickle cell } \\
\text { donor }\end{array}$ & $\begin{array}{l}\text { Percent costaining } \\
\text { of anti-band } 3^{*}\end{array}$ & $\begin{array}{l}\text { Percent costaining } \\
\text { of anti-ankyrin* }\end{array}$ \\
\hline M.K. & 68 & 28 \\
S.Y. & 62 & 32 \\
W.P. & 52 & 36 \\
I.D. & 52 & 32 \\
U.N. & 84 & 58 \\
Average & 63.6 & 37.2 \\
\hline
\end{tabular}

* Permeabilized cells, stained for either band 3 or ankyrin, were viewed by phase contrast microscopy to identify sites of Heinz body binding to the membrane. Care was taken to avoid mistaking endocytotic vesicles for Heinz bodies, the former being frequently observed in sickle cells (1) and appearing more sharply defined than Heinz bodies. Upon localization of each attached Heinz body, the microscope was switched from phase contrast to fluorescence mode and the same site was scored for the presence or absence of a fluorescence spot associated with an elevated density of band 3 or ankyrin. The percent costaining was derived from a sampling of $50 \mathrm{Heinz}$ bodies from each donor. The two donors (T.G. and T.H.) with erythrocytes containing significantly fewer Heinz bodies were not included in the above sampling. inclusion bodies would not be expected to force a redistribution of band 3, at least not until a strong interaction with band 3 had had time to occur. In the opposite situation, where hemichromes first associate with band 3 and then laterally copolymerize into an aggregate, the growth of the Heinz body should parallel the growth of the band 3 cluster. A second reason why some Heinz bodies may not be associated with band 3 clusters is that band 3 does not constitute the sole hemichrome-binding site on the membrane. A substantial number of low-affinity sites were also observed in our earlier hemichrome binding study (16), and native hemoglobin has been reported by others to interact directly with glycophorin and lipid $(6,27,28)$. Thus, a fraction of the hemichromes might be attached to non-band 3 sites that do not influence band 3 distribution. Some of the Heinz body-localized clusters of glycophorin may fall into this category. Thirdly, the absence of anti-band 3 fluorescence at Heinz body sites may occur at sites where anti-band 3 antibodies are physically obstructed from labeling the clustered band 3, perhaps due to a tight adhesion of the ventral membrane surface to the glass slide.

Several laboratories have demonstrated that antibody-coated erythrocytes are selectively trapped and degraded by macrophages in the spleen and liver $(29,30)$. Indeed, antibody-mediated autoimmune hemolytic anemias are well-described pa- 

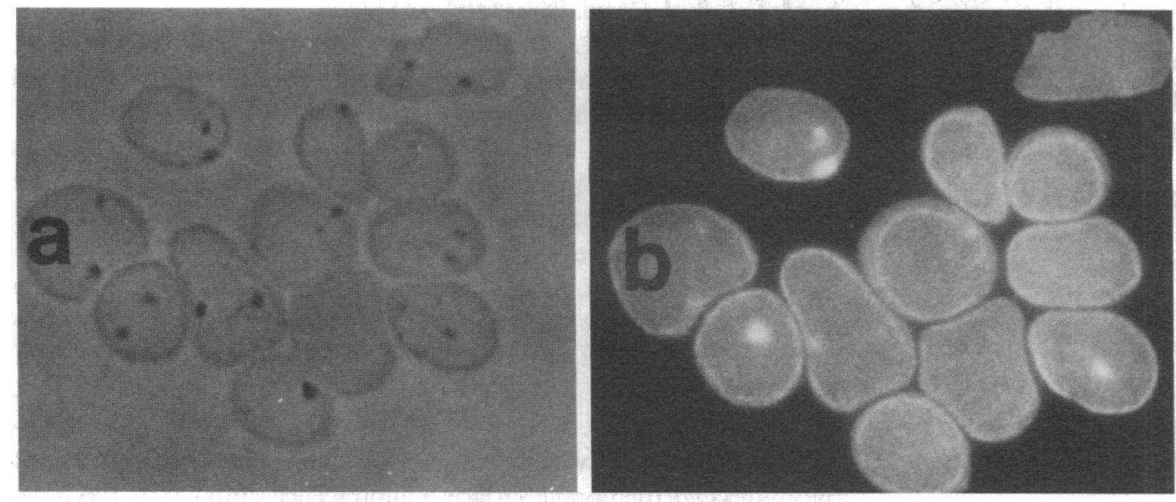

Figure 4. Colocalization of Heinz bodies with clusters of ankyrin in erythrocytes from a sickle hemoglobinopathy patient. Cells were immunostained with antibodies against ankyrin and viewed by phase-contrast $(a)$ or immunofluorescence $(b)$ microscopy.

thologies. Since antibody binding is significantly enhanced in aged cells $(30,31)$, the association of autologous antibodies with an age-dependent cell surface antigen has been proposed as a mechanism of senescent erythrocyte removal. Kay and colleagues have presented evidence that the "senescent antigen" is a proteolysis product of band $3(32,33)$, while Lutz and co-workers believe it is an oligomeric form of the same protein (34). Other labs identify the senescent antigen as desialylated glycophorin (35) or a membrane component bearing a terminal galactose residue $(36,37)$. We have already described a mechanism that can account for the ability of autologous antibodies to recognize aggregated but not dispersed band 3 (17). Here we have demonstrated that the clustered distribution of band 3 can be generated by Heinz body binding to the membrane. While our data were collected only on sickle cells, it is reasonable to expect the same consequences to occur in other erythrocytes containing Heinz bodies (e.g., numerous hemoglobinopathies and senescent red cells). Unfortunately, all attempts to visualize the location of the autologous antibodies on the sickle cell surfaces displayed anti-human IgG staining that was not significantly different from controls. Thus, a more selective and sensitive technique will be required to determine whether clusters of band 3 constitute autologous antibody-binding sites in sickle cells. However, it should be noted that autologous antibody binding is significantly greater on sickle cells than on normal cells (4).

As cells that contain Heinz bodies pass through the spleen, the $\mathrm{Hb}$ aggregate is frequently removed in a process termed "pitting." The Heinz body-promoted clustering of band 3 could provide a mechanism whereby these inclusion body sites might be identified by the spleen. If the pitting mechanism were to involve a concomitant removal of the membrane patch attached to the Heinz body, a selective depletion of band 3 might be anticipated in the filtered cells. Regarding this hypothesis, it should be noted that nonanemic, splenectomized individuals contain the same number of spectrin molecules per red blood cell yet $\approx 20 \%$ more band 3 than nonsplenectomized individuals (38). Furthermore, aged red cells from nonsplenectomized, normal individuals contain $30 \%$ less band 3 per milligram membrane protein than younger cells (39). These two observations indicate that processes that occur in the spleen lead to the selective removal of band 3 relative to spectrin, a cytoskeletal membrane component. It is conceivable that the band 3 clusterHeinz body complex might be recognized and selectively removed by the spleen during pitting, releasing the denser, more rounded erythrocyte to continue to normal function.

To conclude, the numerous abnormalities in sickle cells, e.g., shortened lifespan, elevated $\mathrm{Ca}^{2+}$ content, altered morphology, enhanced levels of surface-bound autologous IgG, abnormal cytoskeletal interactions, altered phospholipid asymmetry, increased oxidant stress, and abnormal cell surface charge distribution (3-14), must ultimately derived from the altered behavior of the $\mathrm{HbS}$ molecule. We have demonstrated that one lesion directly attributable to $\mathrm{HbS}$ is the redistribution of band 3 (and to some extent glycophorin and ankyrin) which accompanies the denaturation of the hemoglobin molecule. It is conceivable that some of the grosser functional and ultrastructural abnormalities of sickle cells may in some way be related to this membrane structural change.
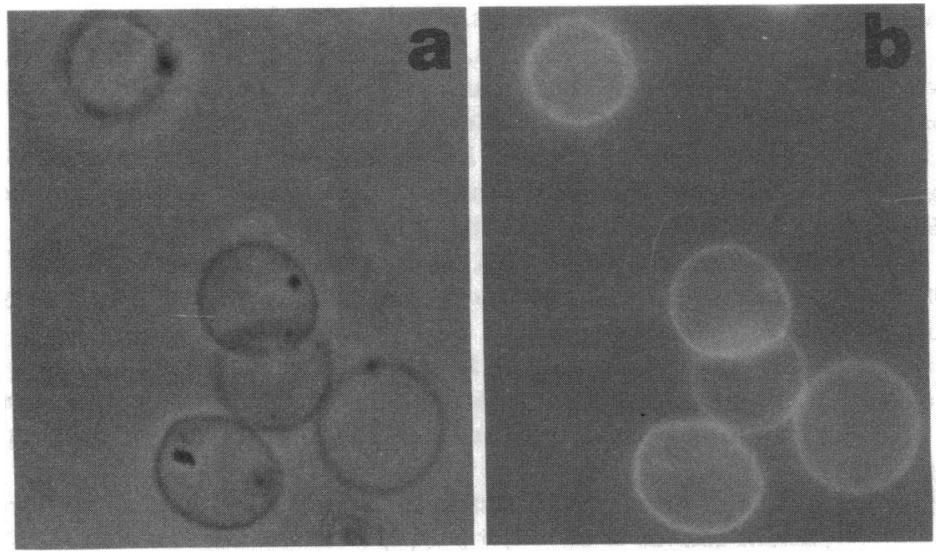

Figure 5. Colocalization of Heinz bodies with clusters of glycophorin. Cells were stained as described in the Methods and viewed by phase-contrast $(a)$ or fluorescence $(b)$ microscopy. 


\section{Acknowledgments}

Antibodies against the cytoplasmic domain of band 3 and ankyrin were prepared in our lab and kindly provided by David Allen and Bernard Thevenin. We wish to thank Sharon Vice for her help in preparing this manuscript.

We wish to acknowledge the National Institutes of Health (GM24417 and HL15168) for partial support of this work

\section{References}

1. Rubin, E., R. A. Schlegel, and P. Williamson. 1986. Endocytosis in sickle erythrocytes: a mechanism for elevated intracellular $\mathrm{Ca}^{2+}$ levels. J. Cell. Physiol. 126:53-59.

2. Bell, A., and K. G. Lofsness. 1986. A photo essay on red cell morphology. J. Med. Tech. 3:87-93.

3. Platt, O. S., J. F. Falcone, and S. E. Lux. 1985. Molecular defect in the sickle erythrocyte skeleton. J. Clin. Invest. 75:266-271.

4. Green, G. A., M. M. Rehn, and V. K. Kalra. 1985. Cell-bound autologous immunoglobulin in erythrocyte subpopulations from patients with sickle cell disease. Blood. 65:1127-1133.

5. Eaton, J. W., E. Berger, J. G. White, and H. S. Jacob. 1978. Calcium-induced damage of Haemoglobin SS and normal erythrocytes. $\mathrm{Br}$. J. Hematol. 38:57-62.

6. Shaklai, N., V. S. Sharma, and H. M. Ranney. 1981. Interaction of sickle cell hemoglobin with erythrocyte membranes. Proc. Natl. Acad. Sci. USA. 78:65-68.

7. McCurdy, P. R. $1969 .{ }^{32} \mathrm{DFP}$ and ${ }^{31} \mathrm{Cr}$ for measurement of red cell life span in abnormal hemoglobin syndromes. Blood. 33:214-224.

8. McCurdy, P. R., and A. S. Sherman. 1978. Irreversibly sickled cells and red cell survival in sickle cell anemia. Am. J. Med. 64:253258.

9. Petz, L. D., P. Yam, L. Wilkinson, G. Garratty, B. Lubin, and W Mentzer. 1984. Increased IgG molecules bound to the surface of red blood cells of patients with sickle cell anemia. Blood. 64:301-304.

10. Fukuda, M., M. N. Fukuda, S. Hakomori, and T. Papayannopoulou. 1981. Anomalous cell surface structure of sickle cell anemia erythrocytes as demonstrated by cell surface labeling and endo- $\beta$-galactosidase treatment. J. Supramol. Struct. Cell. Biochem. 17:289-297.

11. Hebbel, R. P. 1985. Auto-oxidation and a membrane-associated "Fenton reagent": a possible explanation for development of membrane lesions in sickle erythrocytes. Clin. Haematol. 14:129-140.

12. Lux, S. E., K. M. John, and M. J. Karnovsky. 1976. Irreversible deformation of the spectrin-actin lattice in irreversibly sickled cells. $J$. Clin. Invest. 58:955-963.

13. Lubin, B., and D. Chiu. 1981. Abnormalities in membrane phospholipid organization in sickled erythrocytes. J. Clin. Invest. 67:16431649.

14. Hebbel, R. P., O. Yamada, C. F. Moldow, H. S. Jacob, J. G. White, and J. W. Eaton. 1980. Abnormal adherence of sickle erythrocytes to cultured vascular endothelium. Possible mechanism for microvascular occlusion in sickle cell disease. J. Clin. Invest. 65:154-158.

15. Hebbel, R. P., J. W. Eaton, M. Balasingam, and M. H. Steinberg. 1982. Spontaneous oxygen radical generation by sickle erythrocytes. $J$. Clin. Invest. 70:1253-1259.

16. Waugh, S. M., and P. S. Low. 1985. Hemichrome binding to band 3: nucleation of Heinz bodies on the erythrocyte membrane. Biochemistry. 24:34-39.

17. Low, P. S., S. M. Waugh, K. Zinke, and D. Drenckhahn. 1985. The role of hemoglobin denaturation and band 3 clustering in red blood cell aging. Science (Wash. DC). 227:531-533.

18. Bennett, V., and P. J. Stenbuck. 1980. Human erythrocyte ankyrin. J. Biol. Chem. 255:2540-2548.

19. Bennett, U., and P. J. Stenbuck. 1980. Association between ankyrin and the cytoplasmic domain of band 3 isolated from the human erythrocyte membrane. J. Biol. Chem. 255:6424-6432.

20. Appell, K. C., and P. S. Low. 1981. Partial structural character- ization of the cytoplasmic domain of the erythrocyte membrane protein, band 3. J. Biol. Chem. 256:11104-11111.

21. Marchesi, S. L., E. Steers, V. T. Marchesi, and T. W. Tillack. 1970. Physical and chemical properties of a protein isolated from red cell membranes. Biochemistry. 9:50-57.

22. Tyler, J. M., W. R. Hargreaves, and D. Branton. 1979. Purification of two spectrin-binding proteins: biochemical and electron microscopic evidence for site-specific reassociation between spectrin and bands 2.1 and 4.1. Proc. Natl. Acad. Sci. USA. 76:5192-5196.

23. Cherry, R. J., E. A. Nigg, and G. S. Beddard. 1980. Oligosaccharide motion in erythrocyte membranes investigated by picosecond fluorescence polarization and microsecond dichroism of an optical probe. Proc. Natl. Acad. Sci. USA. 77:5899-5903.

24. Low, P. S., W. A. Cramer, G. Abraham, R. Bone, and M. Ferguson-Segall. 1982. Evidence for restricted oligosaccharide mobility at the erythrocyte membrane surface: a fluorescence study. Arch. Biochem. Biophys. 214:675-680.

25. Abraham, G., and P. S. Low. 1980. Covalent labelling of specific membrane carbohydrate residues with fluorescence probes. Biochim. Biophys. Acta. 597:285-291.

26. Hargreaves, W. R., K. N. Giedd, A. Verkleij, and D. Branton. 1980. Reassociation of ankyrin with band 3 in erythrocyte membrane and in lipid vesicles. J. Biol. Chem. 255:11965-11972.

27. Rauenbuehler, P. B., K. A. Cordes, and J. M. Salhany. 1982. Identification of the hemoglobin binding sites on the inner surface of the erythrocyte membrane. Biochim. Biophys. Acta. 692:361-370.

28. Szundi, I., J. G. Szelenyi, J. H. Breuer, and A. Berczi. 1980. Interactions of haemoglobin with erythrocyte membrane phospholipids in monomolecular lipid layers. Biochim. Biophys. Acta. 595:41-46.

29. Gemsa, D., H. H. Fudenburg, and R. Schmid. 1972. Erythrocyte catabolism by macrophages in vitro. Dissociation of phagocytosis and heme oxygenase induction. Trans. Assoc. Am. Physicians. 85:335-343.

30. Kay, M. M. B. 1975. Mechanism of removal of senescent cells by human macrophages in situ. Proc. Natl. Acad. Sci. USA. 72:35213525.

31. Bartosz, G., M. Soszynski, and A. Wasilewski. 1982. Aging of the erythrocyte. XVII. Binding of autologous immunoglobulin G. Mech. Ageing Dev. 20:223-232.

32. Kay, M. M. B., K. Sorensen, P. Wong, and P. Bolton. 1982. Antigenicity, storage, and aging: Physiologic autoantibodies to cell membrane and serum proteins and the senescent cell antigen. Mol. Cell Biochem. 49:65-85.

33. Kay, M. M. B., S. R. Goodman, K. Sorensen, C. F. Whitfield, P. Wong, L. Zaki, and V. Rudloff. 1983. Senescent cell antigen is immunologically related to band 3. Proc. Natl. Acad. Sci. USA. 80:16311635.

34. Lutz, H. U., R. Flepp, and G. Stringaro-Wipf. 1984. Naturally occurring autoantibodies to exoplasmic and cryptic regions of band 3 protein, the major integral membrane protein of human red blood cells. J. Immunol. 133:2610-2618.

35. Alderman, E. M., H. H. Fudenberg, and R. E. Louins. 1981. Isolation and characterization of an age-related antigen present on senescent human red blood cells. Blood. 58:341-349.

36. Galili, U., M. R. Clark, N. Mohandas, E. A. Rachmilewitz, and S. B. Shohet. 1984. The natural anti $\alpha$-galactosyl IgG on red cells in sickle cell disease. Blood. 64(Suppl. 1):48a. (Abstr.)

37. Galili, U., A. Korkesh, I. Kahane, and E. A. Rachmilewitz. 1983. Demonstration of a natural antigalactosyl IgG antibody on thalassemic red blood cells. Blood. 61:1258.

38. Agre, P., J. F. Casella, W. H. Zinkham, C. McMillan, and V. Bennett. 1985. Partial deficiency of erythrocyte spectrin in hereditary spherocytosis. Nature (Lond.). 314:380-383.

39. Miyahara, K., and M. J. Spiro. 1984. Nonuniform loss of membrane glycoconjugates during in vivo aging of human erythrocytes: studies of normal and diabetic red cell saccharides. Arch. Biochem. Biophys. 232:310-322.

40. Fairbanks, G., T. L. Steck, and D. F. H. Wallach. 1971. Electrophoretic analysis of the major polypeptides of the human erythrocyte membrane. Biochemistry 10:2606-2617. 